\title{
Analyse Genotypique De La Contribution De La Matiere Assimilée Du Pedoncule Dans Le Remplissage Du Grain Chez Le Ble Dur (Triticum Durum D.E.S.F.)
}

\author{
Linda Kahali, MA \\ Abdelhamid Djekoun, Prof
}

Laboratoire de Génétique, Biochimie et Biotechnologie Végétale. Faculté des Sciences de la Nature et de la Vie. Université Constantine1, Ain El bey,

Algérie

doi: 10.19044/esj.2016.v12n18p151 URL:http://dx.doi.org/10.19044/esj.2016.v12n18p151

\begin{abstract}
In a part of study on morpho-physiological characterization in durum wheat; we were interested in quantifying the stem dry matter produced after anthesis and its contribution in the grain filling. When the climatic conditions are unfavorable ; the photosynthetic activity of the post-anthesis is reduced. The plant then uses the assimilates stored in the stem to increase its filling rate of grain. Thus, the behavior of four varieties of durum wheat is analyzed in the field using different parameters: (i) relative water content (RWC) of the flag leaf (ii) dry weight development of the grains (iii) peduncle assimilated dry matter contribution in the grain filling. The leaf water status, appreciated by measuring the RWC, is an indicator of the photosynthetic apparatus functioning. The measured values showed a significant difference between two agricultural companions. The results also showed a genotypic difference in the dry matter accumulation in the grains, mainly related to its filling speed. The accumulation of assimilates in the peduncle during the days following flowering, constitutes an advantage of reserves in dry matter before installation of water deficit. Indeed, it affects production by decreasing the photosynthetic activity. In conclusion, this experiment has highlighted two types of grain yield elaboration of cultivated wheat in the post-anthesis water deficit conditions.
\end{abstract}

Keywords : Peduncle assimilates, water deficit, grain filling, relative water content, Triticum durum

\section{Résumé}

Dans le cadre d'une étude portant sur caractérisation morphophysiologique chez le blé dur ; nous nous sommes intéressés à la 
quantification de la matière sèche de la tige produite après anthèse et sa part de contribution dans le remplissage du grain. Lorsque les conditions climatiques sont contraignantes; l'activité photosynthétique de la postanthèse est réduite. La plante fait alors appel aux assimilats stockés dans la tige pour accroître son taux de remplissage des grains. Ainsi, le comportement de quatre variétés de blé dur est analysé en plein champ, par différents paramètres : (i) teneur relative en eau (TRE) de la feuille étendard (ii) évolution du poids sec des grains (iii) estimation de la contribution de la matière sèche assimilée du pédoncule dans le remplissage du grain. L’état hydrique de la feuille appréciée par la mesure de la TRE, est un indicateur du fonctionnement de l'appareil photosynthétique. Les valeurs mesurées montrent une différence significative entre les deux compagnes agricoles. Les résultats montrent également une différence génotypique dans l'accumulation de matière sèche dans les grains, principalement reliée à la vitesse de remplissage de celle-ci. L'accumulation des assimilats dans le pédoncule au cours des jours qui suivent la floraison, constitue un avantage de réserves en matière sèche ; avant que ne s’installe le déficit hydrique. En effet, il affecte la production par diminution de l'activité photosynthétique. En conclusion, cette expérimentation a permis de mettre en évidence deux types d'élaboration du rendement en grains du blé dur cultivé en conditions de déficit hydrique post- floraison.

Mots clés : Assimilats du pédoncule, déficit hydrique, remplissage du grain, teneur relative en eau, Triticum durum

\section{Introduction}

La matière sèche du grain de blé dur résulte de la contribution de trois sources ; trouvant leur origine dans le fonctionnement photosynthétique de trois types d'organes assurant le remplissage du grain: les feuilles, la tige et les parties vertes de l’épi (Sanchez Bragado et al., 2014).

Le rendement en grains chez le blé est le résultat des assimilats produits au cours de la vie de la plante. Il est départagé en trois composantes :

- $\quad$ La matière sèche produite après anthèse et transférée directement aux grains.

- $\quad$ La matière sèche produite avant anthèse et remobilisée vers le grain durant la période de remplissage du grain.

- $\quad$ La matière sèche produite après anthèse mais stockée temporairement dans les organes végétatifs, avant d’être remobilisée vers les grains (Pheloung et Siddique, 1991).

La tige du blé constitue la principale localisation de matière sèche ultérieurement remobilisée. Plusieurs auteurs (Gallagher et al., 1977 et Bidinger et al., 1977) ont montré une contribution significative des assimilats 
produits durant la phase pré-anthèse. Ils ont indiqué qu’à peu près $50 \%$ des photosynthétats produits après anthèse sont d'abord stockés pour quelques jours au niveau de la tige avant d'être transférés vers le grain.

Gate et al. (1992) montrent que la contribution de la tige s'accroît avec l'installation du déficit hydrique, lors de la phase de remplissage du grain. Cette contribution augmente substantiellement dans ces conditions pour accroître le taux de remplissage du grain (Bahlouli et al., 2008).

Ce travail entre dans le cadre d'une étude de caractérisation morpho-physiologique de quatre variétés de blé dur différant par la taille du chaume. Nous nous sommes intéressés à la quantification de la matière sèche de la tige produite avant et après anthèse et sa part de contribution dans le rendement en grains chez quatre variétés de blé dur cultivées en plein champ.

\section{Matériel et méthodes \\ Matériel}

Les essais ont porté sur quatre variétés de blé Triticum durum. Deux variétés introduites caractérisées par une paille relativement courte (Waha et Vitron) et deux variétés locales hautes (Hedba3 et Bidi 17). Ces génotypes supposés de tolérance différente vis-à-vis de la sécheresse. Elles ont été choisies dans le cadre d'un programme national de développement agricole (P.N.D.A) (Tableau 1) :

Tableau 1 : Liste des variétés introduites dans l'étude morpho-physiologique.

\begin{tabular}{|c|c|c|c|}
\hline Variétés & Origine & source & Caractéristiques \\
\hline Waha & CIMMYT, ICARDA & I.T.G.C. & $\begin{array}{c}\text { Précoce, paille courte, bonne } \\
\text { productivité }\end{array}$ \\
\hline Vitron & CIMMYT, ICARDA & I.T.G.C. & $\begin{array}{c}\text { Semi précoce, paille moyenne, } \\
\text { productive }\end{array}$ \\
\hline Hedba3 & ALGERIE & I.T.G.C. & Paille haute, productivité moyenne \\
\hline Bidi17 & ALGERIE & I.T.G.C. & $\begin{array}{c}\text { Semi tardive, paille haute, } \\
\text { productivité moyenne }\end{array}$ \\
\hline
\end{tabular}

I.T.G.C : Institut Technique des Grandes Cultures

CIMMYT : Centro Internacional de Mejoramiento de Maiz y Trigo

\section{Méthodes}

\section{Conduite de l'essai}

L'expérimentation a été conduite sur deux compagnes agricoles successives : 2000/2001 et 2001/2002 à la Station Expérimentale de l'Institut Technique des Grandes Cultures (ITGC) d'El Khroub située à $14 \mathrm{Km}$ au sud de Constantine. Le site se trouve à une altitude de $640 \mathrm{~m}$, à la latitude $6^{\circ} 67^{\prime}$ et une longitude de $36^{\circ}, 25^{\prime}$ nord. La zone représentative des régions semiarides de l'Algérie orientale, est froide l'hiver avec un été chaud et sec.

Les données pluviométriques, provenant du dépouillement des fiches de relevés effectués sur une période de 25 ans (1970/71-1994/95) montrent 
que la moyenne (de septembre à juin) est de 506 mm. Les deux compagnes agricoles sont caractérisées par une pluviométrie très faible par rapport à cette moyenne, soit respectivement $288.8 \mathrm{~mm}$ et $263.1 \mathrm{~mm}$. Cependant les mois d'Avril et Mai (période correspondant à la phase floraison- remplissage du grain) ont été plus pluvieux durant la compagne agricole 2001, avec une moyenne de $32.8 \mathrm{~mm}$ contre $11 \mathrm{~mm}$ seulement l'année suivante.

\section{Paramètres étudiés}

Les paramètres étudiés ont été mesurés aux stades les plus caractéristiques du développement de la plante; du stade floraison au stade maturité au champ.

\section{Teneur relative en eau de la feuille étendard}

La teneur relative en eau (TRE) est mesurée selon la formule de Barrs, 1968

\section{TRE (\%) = [(PF - PS) / (PT - PS) $]$ x 100}

PF : poids frais de la feuille, déterminé immédiatement après prélevement.

PS : poids sec, mesurée après séchage à l'étuve à $80^{\circ} \mathrm{C}$ pendant 24 heures.

PT : poids de turgescence, mesuré après avoir placé les feuilles dans des tubes contenant de l'eau distillée, à l'obscurité pendant 24 heures. La pesée est réalisée après un essuyage soigneux de la feuille avec du papier filtre.

\section{Extraction de la matière sèche soluble dans l'éthanol (MSSE)}

La matière sèche soluble dans l'éthanol de la tige est déterminée par la méthode de Blacklow et al., (1984). Elle est calculée par l'équation : MSSE = MST - MSIE.

Les tiges sectionnées à partir du dernier nœud et séchées à l'étuve pendant 24 heures à $80^{\circ} \mathrm{C}$ sont coupées en petits morceaux de 3 à $4 \mathrm{~mm}$.

On pèse100 mg des sections de tige (MST) et on les place dans des tubes en verre. On ajoute $10 \mathrm{ml}$ d'éthanol à 70\%. On couvre à l'aide de billes en verre pour minimiser l'évaporation de l'alcool et incube pendant deux heures au bain- marie à $80^{\circ} \mathrm{C}$ avec agitation périodique. On prélève le surnagent à l'aide d'une pipette pasteur et on fait subir au résidu la même opération encore deux fois. Le résidu final est par la suite placé à l'étuve à $80^{\circ} \mathrm{C}$ pendant 24 heures puis pesé, c'est ce qui représente la matière sèche insoluble dans l'éthanol (MSIE).

\section{Contribution (en \%) de la réserve de la tige dans le remplissage du grain}

La contribution de la tige est exprimée comme étant le rapport entre la diminution de cette réserve et le poids final des grains par épi, au stade maturité physiologique (Pheloung et Siddique, 1991). 


\section{Analyse Statistique}

L'interprétation des résultats de la TRE (moyenne de cinq répétitions) est réalisée par une analyse de variance à deux facteurs. Elle est suivie par le test de classement des moyennes, Newman Keuils (SNK) par le logiciel XL Stat Version 2010.

Les graphiques et les histogrammes sont effectués par le logiciel Exel

\section{Résultats et discussion}

\section{Evolution de la MSSE du pédoncule}

L'évolution générale de la MSSE est similaire chez tous les génotypes évalués. Elle passe par deux phases distinctes : phase ascendante et phase descendante (Fig. 1).

Dans nos conditions expérimentales, nous constatons que la MSSE augmente pour atteindre une valeur maximale à partir du $10^{\text {ème }}$ jour ou du $18^{\text {eme }}$ jour après l'anthèse (Fig. 1). Ceci, selon le génotype étudié et également selon les résultats des deux compagnes agricoles

Selon l'expérimentation 2001 (Fig. 1A), une différence de comportement est constatée. La valeur maximale est atteinte au $14^{\text {ème }}$ jour chez les variétés Waha et Vitron; au $10^{\text {ème }}$ jour chez Bidi17, par contre, elle est atteinte par Hedba 3 au $18^{\text {ème }}$ jour. En travaillant sur le blé d’hiver et le blé de printemps, Blacklow et al. (1984) ont pu montrer une accumulation maximale de MSSE au niveau de la tige au bout de 18 jours.

La valeur de MSSE la plus importante est retrouvée chez la variété Hedba3 $(0.26 \mathrm{~g})$, pour le reste des génotypes, les valeurs se rapprochent et varient entre $0.21 \mathrm{~g}$ et $0.22 \mathrm{~g}$.

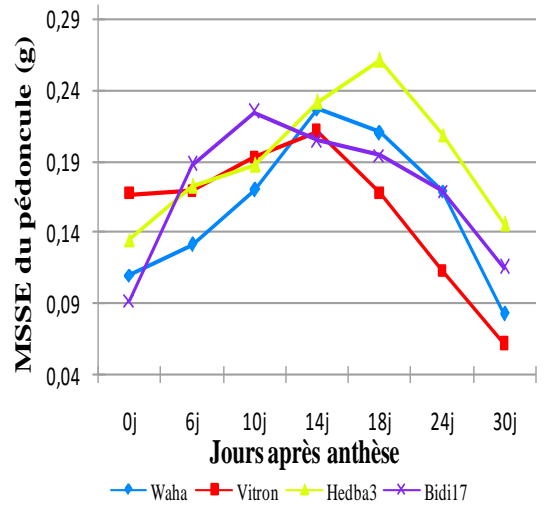

Fig. 1.

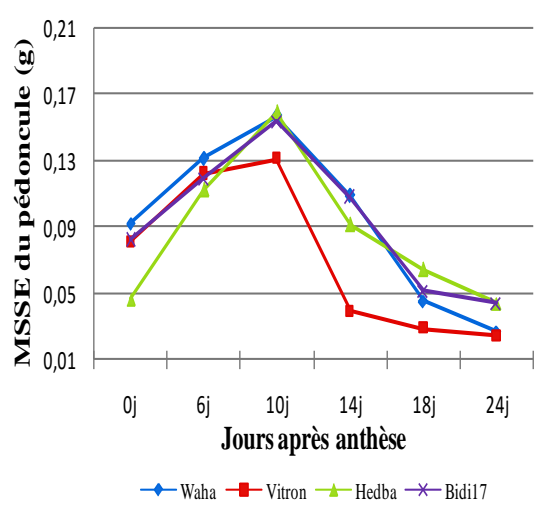

A
B 
Evolution de la MSSE durant la phase post anthèse chez quatre variétés de blé dur cultivées en plein champ. A : Compagne 2001, B : Compagne 2002

L’impact des conditions climatiques, qui ont sévi l'année suivante (2002), est bien marqué (Fig. 1B). En effet, on observe non seulement un changement notable sur la durée d'accumulation de matière sèche, mais aussi sur sa quantité et ce, chez l'ensemble des génotypes étudiés.

La durée d'accumulation de MSSE s’étale seulement sur 10 jours pour l'ensemble des variétés. En parallèle, la quantité de MSSE accumulée a significativement diminué, par rapport à l’année 2001. Elle varie entre $0.13 \mathrm{~g}$ et $0.15 \mathrm{~g}$.

En outre, nos résultats montrent que la MSSE accumulée dans le pédoncule est très liée à sa longueur; ce qui explique la forte corrélation entre ces deux paramètres $(r=0.94)$. Le rôle du pédoncule s'expliquerait par la quantité d’assimilats photosynthétiques stockées dans cette partie de la plante et susceptibles d'être transférée vers le grain. Les travaux de Nachit et Ketata (1991) et ceux de Loss et Siddique (1994) montrent que la capacité de stockage des réserves dans la tige, est en fonction de la hauteur de la plante et de la taille du col de l'épi.

La MSSE décline avec la matière sèche de la tige. Ce phénomène s'explique par la translocation des assimilats vers le grain au cours de son remplissage. La diminution de la MSSE au cours de cette phase, pour laquelle nous attribuons le terme de "vidange" n'est pas importante durant les quatre jours qui suivent le pic maximale.

Les variétés waha, Hedba3 et Bidi17 accusent des taux de diminution variant entre $5.28 \%$ à $9.8 \%$ contre un taux plus élevé (20.63 \%) pour Vitron (Fig. 1A).

Quatre semaines après l'anthèse, le taux de diminution de la MSSE fait apparaître des différences variétales. Le taux de diminution est relativement faible chez les deux variétés Hedba3 et Bidi17, avec des valeurs respectives de $44.2 \%$ et $48.59 \%$. Contrairement, La diminution de la MSSE est plus importante chez les autres variétés Waha et vitron, avec des taux respectifs de $63.45 \%$ et $70.73 \%$. D’où probablement, une meilleure remobilisation des réserves de la tige vers le remplissage du grain. Et donc, une vitesse d'accumulation de la matière sèche (g/jour) dans le grain plus élevée chez ces deux variétés.

Les résultats de l’année 2002, montrent une situation différente par rapport à l'année précédente mais globalement analogue pour l'ensemble des génotypes étudiés (Fig. 1B). Deux semaines après l'accumulation maximale de la MSSE, Vitron et Waha montrent un taux de diminution supérieur à 81 \% contre plus de 71\% pour les deux autres variétés. 


\section{Evolution du poids sec moyen des grains par épi}

La forme générale de l'évolution du poids sec des grains par épi est similaire chez l'ensemble des génotypes utilisés. Elle passe par deux phases (Fig.2) :

- $\quad$ une première phase de remplissage rapide, de durée variable deux semaines et moins selon l'année.

- $\quad$ une deuxième phase caractérisée par un faible taux d'accumulation de matière sèche au niveau du grain.

La différence génotypique dans l'accumulation de la matière sèche dans les grains est principalement liée, à la vitesse de remplissage de celle-ci. Selon Triboï (1990), la durée de remplissage représente le nombre de jours permettant d'atteindre un poids du grain maximum et stable. Alors que la vitesse de remplissage représente la quantité de matière sèche accumulée dans le grain par jour par épi.

Au cours de l'expérimentation 2001 (Fig.2A), cette vitesse varie entre $4.39 .10^{-2} \mathrm{~g} / \mathrm{j} /$ épi à $5.27 .10^{-2} \mathrm{~g} / \mathrm{j} /$ épi respectivement pour Hedba3 et Bidi17. Par contre les deux variétés Vitron et Waha se distinguent par des vitesses d'accumulation nettement plus élevées. Avec respectivement $7,26.10^{-2}$ et $7,59.10^{-2} \mathrm{~g} / \mathrm{j}$ /épi et pour une durée de remplissage équivalente. Cette forte activité de remplissage, est probablement liée à une capacité photosynthétique favorable et donc une accumulation post-floraison de matière sèche relativement plus importante pour ces deux génotypes.

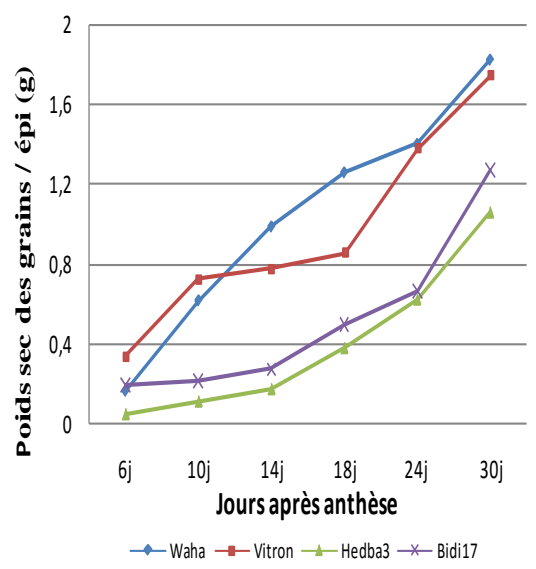

A

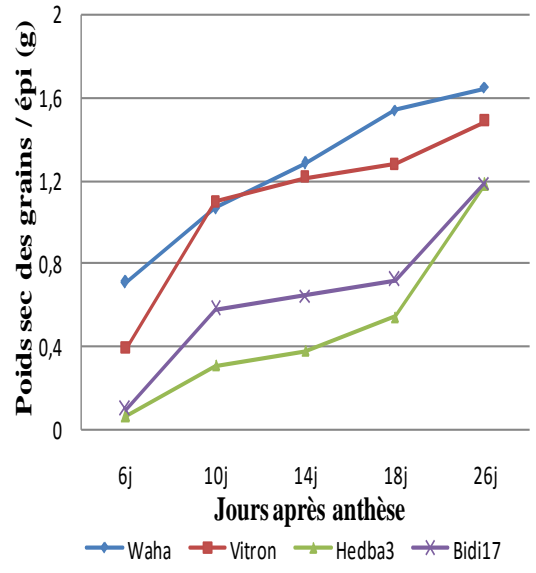

Fig. 2. 
Evolution du poids sec des grains par épi chez quatre variétés de blé dur cultivées en plein champ. A : Compagne 2001, B : Compagne 2002

L’année 2002 se caractérise par une sécheresse dès la montaison et par un remplissage en conditions très échaudantes. Parallèlement au stress hydrique lié aux insuffisances pluviales et à leur irrégularité ; les températures élevées en zone semi arides peuvent constituer, elles aussi, une contrainte climatique sérieuse pour la production céréalière (Zouaoui et al., 2007). Les fortes températures au cours du remplissage du grain provoquent l'arrêt de la migration des réserves des feuilles et de la tige vers le grain : c'est l'échaudage du grain. Nous avons en parallèle noté une absence de grains dans les épis, au cours des observations. Les conditions de forte sécheresse et de hautes températures autours de la floraison, ont un effet direct sur le processus de fécondation. Ils causeraient la stérilité du pollen (Bingham, 1966 ; Debaeke et al., 1996).

Cependant au cours de cette année, bien qu'exceptionnelle, les quatre génotypes ont globalement un comportement identique à l'année précédente (Fig. 2B). Effectivement, la vitesse de remplissage est toujours élevée chez Vitron et waha avec respectivement, $7.43 .10^{-2} \mathrm{~g} / \mathrm{j}$ /épi et $8.23 .10^{-2} \mathrm{~g} / \mathrm{j}$ /épi. Une vitesse de remplissage élevée est indicatrice des effets du stress hydrique (Sofield et al., 1977). Chez les deux autres variétés, la vitesse de remplissage est plus faible mais analogue de l'ordre de $5.9 .10^{-2} \mathrm{~g} / \mathrm{j}$ /épi. Cependant, la variété Hedba3 semble accumuler la matière sèche à un rythme plus long. Chez celle-ci, l'évolution du poids sec des grains se caractérise par un faible taux d'accumulation de matière sèche durant la phase linéaire de remplissage. Probablement dû à une faible migration des assimilats stockés dans les différents organes de la plante, contribuant au remplissage du grain.

Deux contraintes ont marqué l'année 2002, la faiblesse des précipitations et leur distribution aléatoire. Le manque d'eau au cours de la phase du remplissage du grain a affecté la production des assimilats par manque d'activité photosynthétique de fin de cycle (Blum et al., 1994). Un stress hydrique durant la période de remplissage du grain réduit la photosynthèse et raccourcit la période de remplissage du grain. Ceci concorde avec les travaux de Yang et Zhang (2006).

\section{Taux de contribution de la MSSE du pédoncule dans le remplissage du grain}

Une partie importante de la croissance des grains serait due à l'activité photosynthétique des feuilles en période post- floraison. En effet, le taux de migration des substances carbonées des feuilles vers les grains augmente rapidement à partir de l'anthèse. Ainsi, la répartition de la matière sèche (MS) des grains (Fig. 3) entre la phase d'augmentation de la MSSE, qu'on appellera phase ascendante et de sa diminution ou phase descendante, 
montre que $16.64 \%$ à 54 \% (résultats de l'année 2001) de MS est accumulée durant la phase ascendante (Fig.3A). Ce qui montre une variabilité génotypique dans l'accumulation de MSSE au niveau des grains au cours de cette première phase. La variété Bidi17 accumule la plus faible quantité de MS. Contrairement, la variété Waha présente la valeur la plus élevée. Ce qui traduit probablement un poids sec des grains plus élevé chez cette variété.

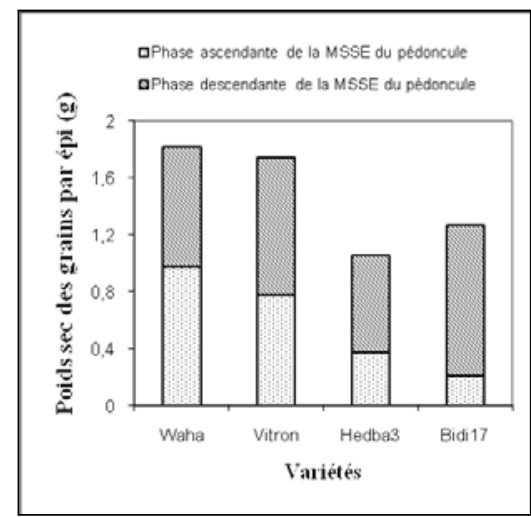

A

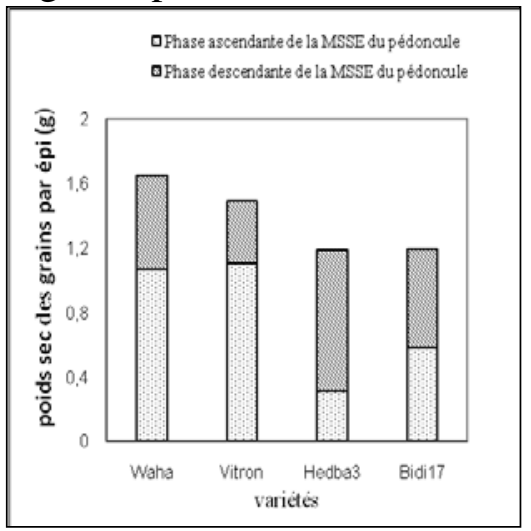

B

Fig.3. Evolution du poids sec des grains durant la phase post-anthèse A : Compagne 2001, B : Compagne 2002

Les observations de l'essai 2002 (Fig.3B) laissent toujours dégager cette variabilité mais avec un comportement différent de l'année précédente. Elle est illustrée par, l'accumulation de la MS qui augmente au cours de cette première phase ; chez l'ensemble des variétés, avec des taux de $49 \%$ à $74 \%$. Excepté Hedba3 qui affiche le taux le plus faible $26.12 \%$. Cette variété représente aussi le plus faible poids sec des grains par épi.

Ainsi, nous enregistrons une corrélation positive entre la quantité de MS accumulée dans les grains pendant cette phase et le poids sec final des grains ( $r=0.89$ en 2001 et $r=0.91$ en 2002).

La contribution de la MSSE du pédoncule (exprimée en \%) dans la matière sèche accumulée dans les grains durant la phase descendante (ou encore vidange) ; montre des valeurs assez proches (Fig. 4A). Elles varient entre $15.36 \%$ à $17.15 \%$ pour l'ensemble des variétés, excepté la variété Bidi17 qui accuse le taux le plus faible (10.32\%). 


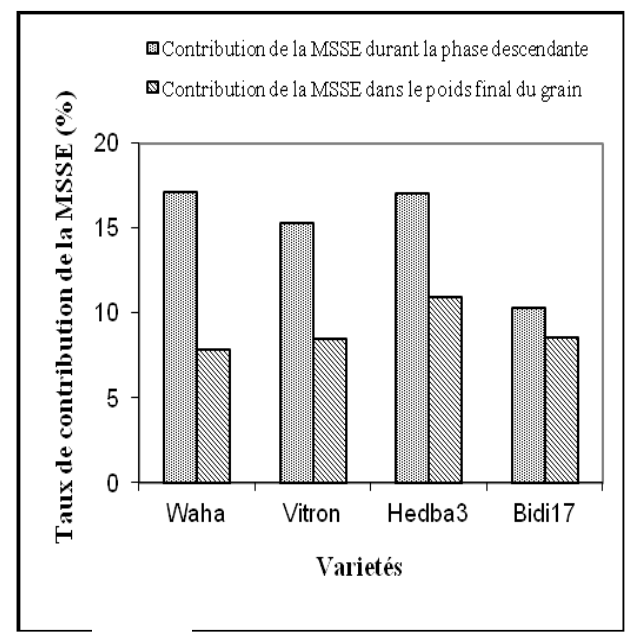

A

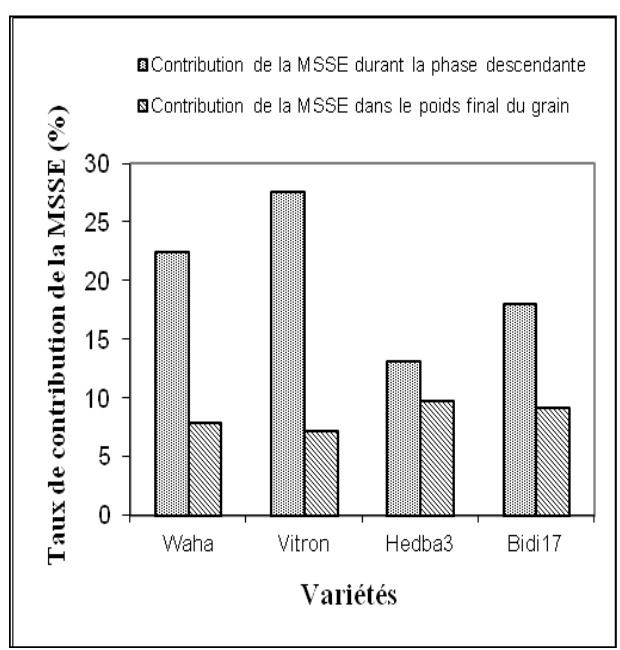

B

Fig. 4. Taux de contribution de la MSSE dans le remplissage du grain.

A : Compagne 2001, B : Compagne 2002

L'effet année est significatif. Au cours de la compagne 2002, la contribution de MSSE (Fig.4B) diminue pour la variété Hedba3 (13.18\%) pour cette même phase contre un taux, qui augmente chez le reste des variétés (18\% à 27.5\%). Ceci, peut être associé à un transfert de grandes quantités des assimilats stockés dans la tige. La contribution du pédoncule est cruciale durant les étapes finales du remplissage du grain (Kong et al., 2010).

La part des réserves transférée est relativement marginale en bonnes années pour devenir importante en conditions de déficit hydrique; lorsque l'appareil photosynthétique est fortement affecté (Triboï et al., 1985b ; Kaubauch et thome, 1989 ; Bahlouli et al., 2008). Mais elle varie selon la variété étudiée (Kahali, 1998 et El Fakhri et al., 2012). Lorsque les conditions climatiques sont contraignantes, l'activité photosynthétique de la post-anthèse est réduite, ce qui augmenterait la remobilisation des carbohydrates non structurés des tissus végétatifs vers le grain (Yang et zang, 2006). Cette mobilisation des réserves est une source de contribution importante dans le remplissage du grain (Pandey et al., 2002).

Par ailleurs, la contribution des réserves de la tige dans le remplissage des grains (exprimé par rapport au poids sec final des grains par épi) représente la quantité remobilisée vers les grains. Elle est plus ou moins identique (7.89 \% à $8.6 \%$ ) pour l'ensemble des variétés (Fig. 4A). La variété Hedba3 a le taux le plus élevé (10.98\%). Ce taux reste globalement identique l'année qui suit (Fig.4B). Cependant, on note une légère augmentation chez Bidi17 contre une diminution chez Hedba3; sachant 
qu’elle affiche toujours la valeur la plus élevée. Les variétés à chaume long montrent une remobilisation importante des assimilats stockés dans le pédoncule (Masghoudi Moud et islami, 2011). Le pédoncule représente un site de stockage des réserves en vue d'un meilleur remplissage du grain. Les sucres accumulés proviennent de l'hydrolyse des réserves d'amidon des racines (Bahlouli et al., 2008)

En outre, les variétés Waha et Vitron avec leurs faibles taux de contribution de la tige à l'égard des variétés Hedba3 et Bidi17, présentent un poids sec des grains par épi relativement plus élevés. Ceci peut être lié à une meilleure utilisation des assimilats stockés ou provenant de l'activité photosynthétique instantanée de la feuille étendard.

Dans cette optique, nous avons essayé d'aborder cette question, en traitant la relation qui pourrait exister entre l'état hydrique des feuilles et le niveau de remplissage des grains par épi.

\section{Etat hydrique de la plante et sa relation avec le remplissage du grain}

La caractérisation du statut hydrique d'une plante pourrait passer par la seule évolution de la teneur relative en eau. En effet, Sinclair et Ludlow (1985) considèrent le critère TRE comme un meilleur indicateur de l'état hydrique des tissus comparé aux potentiels: hydrique, osmotique ou de turgescence. Cependant, il est important de souligner que l'évolution de ce paramètre, rend compte des variations de la quantité d'eau dans les tissus des plantes (Aissa et Radhouane, 2014) particulièrement en situation de contrainte hydrique.

L'évolution de l'état hydrique est appréciée par la mesure de la TRE de la feuille étendard.

Lors de la première compagne (2001) ; la TRE, à l'anthèse est très élevée. Elle est globalement peu différente entre variétés (Fig. 5A). Une semaine après l'anthèse, une légère baisse est notée chez les quatre variétés.

$\mathrm{Au}$ cours du dessèchement progressif du sol, des différences de comportement hydriques sont notées. En effet, deux semaines après la floraison, nous constatons une diminution de la TRE allant de $14 \%$ à $16 \%$ chez les variétés Waha, Vitron et Hedba3. Par contre, elle est d'environ $11 \%$ pour la variété Bidi17, qui maintient une TRE relativement plus importante (82.08\%). Avant la sénescence totale de la feuille étendard, les feuilles maintiennent une quantité d'eau relativement appréciable. Le taux de diminution est de $20 \%$ chez les variétés Vitron et Hedba3 mais plus élevé chez les deux autres Bidi17 et Waha (20.27\%, $26.11 \%$ respectivement). Selon Cornic (1994) une diminution de 30\% du contenu en eau des feuilles affecte significativement la photosynthèse. 


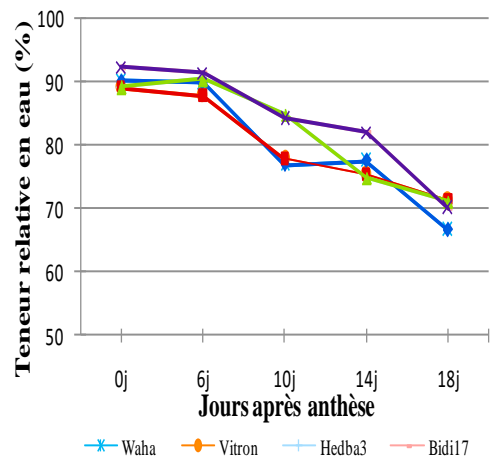

A

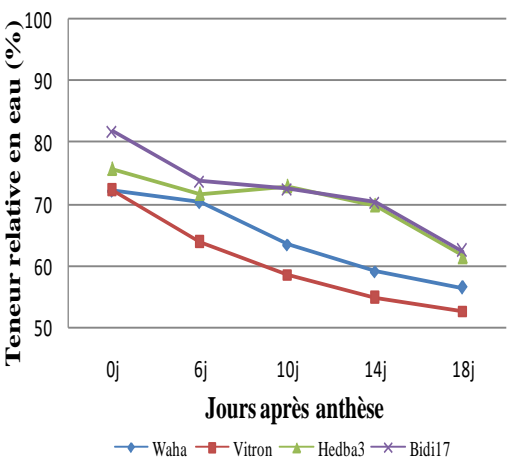

B

Fig.5. Evolution de la teneur relative en eau ue la feuille étendard A : Compagne 2001, B : Compagne 2002

Le déficit hydrique de forte intensité représenté par l'année 2002 (Fig.5B) fait apparaître des valeurs de TRE plus faibles par rapport à l'année précédente. Ainsi, l'analyse de la variance montre une différence hautement significative quant aux résultats des deux années pour ce paramètre, au seuil 5\% (Tab.2). Par contre, elle ne reflète aucune différence significative entre les variétés étudiées au sein de la même compagne pour les deux années 2001et 2002 successivement.

Tableau $n^{\circ} 2$ : Analyse de variance pour la TRE

\begin{tabular}{cccccc}
\hline Source & ddl & Somme des carrés & Carré moyen & F de Fisher & Pr $>$ F \\
\hline Compagne & 1 & 2223,081 & 2223,081 & 35,240 & $<0,0001$ \\
Variété & 3 & 378,686 & 126,229 & 2,001 & 0,134 \\
Compagne*Variété & 3 & 111,181 & 37,060 & 0,587 & 0,628 \\
\hline
\end{tabular}

Le test Newman Keuils (SNK) au seuil 5\% classe le facteur compagne en deux groupes :

A $>$ B $\Leftrightarrow$ Compagne 2001>compagne $2002 \Leftrightarrow 81.67>66.76$

Le même test (SNK) classe les quatre variétés étudiées en seul groupe pour les deux compagnes :

$$
\text { 2001: Bidi17 }<\quad \text { Hedba } 3<\quad \text { Vitron }<\text { Waha }
$$

$\Leftrightarrow 84.05 \angle 82.13<80.30<80.21$

2002 : Bidi17 $<$ Hedba $3<$ Vitron $\angle$ Waha $\Leftrightarrow 72.11<70.28<64.29$ $\angle 60.36$

Au stade de l'anthèse $(0 \mathrm{j})$, nous avons noté une différence variétale vis-à-vis de la TRE. Elle varie entre $72,2 \%$ et $81,81 \%$ au cours de cette 
année (Fig.5B). Cependant, il faut signaler que ces valeurs étaient aux alentours de $90 \%$ en 2001 au même stade de mesure (Fig.5A)

La sécheresse entraîne une diminution du potentiel hydrique du sol, et ceci s'explique par une perte importante de la turgescence au niveau de la plante.

Avant la sénescence de la feuille étendard, les valeurs de la TRE sont faibles par rapport à celles enregistrées l'année précédente (2001). Elles sont aux alentours de $62 \%$ chez les variétés Bidi17 et Hedba3; proche de $57 \%$ pour la variété Waha. Par contre la variété Vitron, affiche un niveau encore plus faible, inférieure à $53 \%$. Sofield et al. (1988) notent que la teneur en eau diminue lorsque le stress hydrique augmente.

Ces valeurs relativement élevées juste avant la sénescence totale de la feuille drapeau (vue les conditions climatiques contraignantes) peuvent ou non traduire une forte production de grains par épi. La variété Hedba3 avec une TRE de $62 \%$, présente une faible production de grain par épi (29.7) alors que Waha pour une valeur proche de cette dernière $(\approx 57 \%)$ a un nombre en grains par épi plus élevé (44.1). On constate donc que la sensibilité au déficit hydrique pour l'élaboration du rendement n’est pas toujours liée à une teneur élevée en eau (Lewicki, 1993). La variété Waha présente un nombre de grains par épi élevé et le poids sec de grain par épi le plus élevé, les deux années consécutivement. Selon Slama et al. (2005), de telles variétés se distinguent des autres par une conductance et une densité stomatique plus élevées au niveau de la barbe et de la feuille drapeau.

Par ailleurs, la TRE diminue au cours de la vidange de la tige (phase descendante de la MSSE); ce qui correspond à une phase intense du remplissage du grain. Les résultats des deux conduites agricoles (Fig. 6) montrent des corrélations négatives (respectivement $r=-0,61$ et $r=-0,85$ ) entre l'état hydrique de la feuille étendard et le poids sec des grains durant la

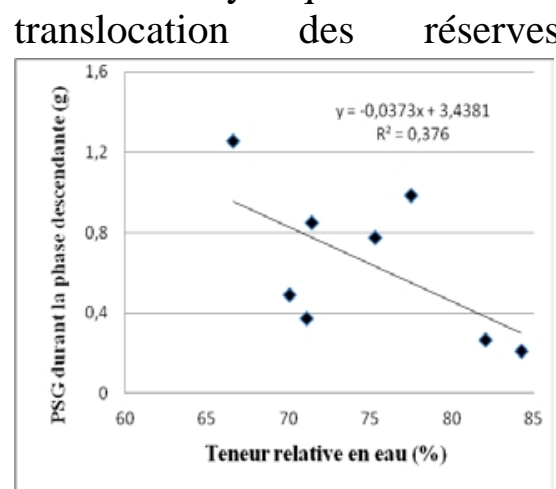

A

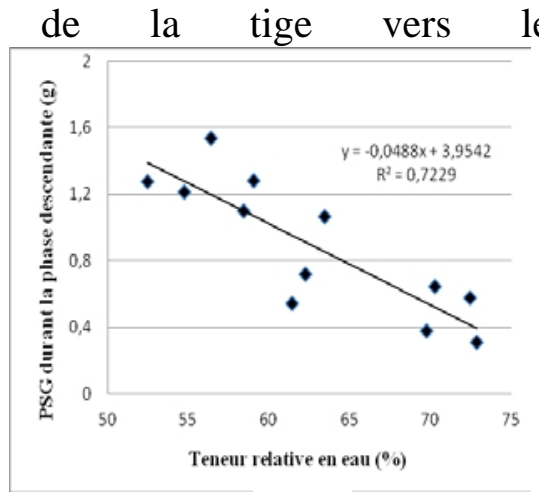

B

Fig. 6. Relation TRE et poids sec des grains (PSG) durant la phase de vidange du pédoncule. A : Compagne 2001, B : Compagne 2002 


\section{Conclusion}

A l'anthèse, la quantité de matière sèche produite doit être suffisante pour assurer le remplissage du grain et donc un rendement en grains acceptable. Ainsi, au cours de cette expérimentation, nous avons pu mettre en évidence chez les variétés étudiées, deux types de stratégies d’élaboration du rendement en grain du blé dur cultivé en plein champ sous des conditions de déficit hydrique post- floraison :

- la première est basée sur les capacités de stockage de MSSE dans la tige et sa remobilisation vers le grain (cas de Hedba3).

- la deuxième est basée sur la capacité de la plante à maintenir une activité photosynthétique malgré le déficit hydrique (cas de Waha).

La capacité de stockage des réserves photosynthétiques dans la tige n'est pas sans importance, en ce qui concerne la problématique de la tolérance à la sécheresse. Cette accumulation constitue un avantage en réserves de MSSE. Elle est remobilisée vers les grains lorsqu'il y a déficit en assimilats suite à la baisse ou à l'absence de la photosynthèse.

L'autre aspect qui contrôle l'élaboration du rendement en grains du blé dur en conditions de déficit hydrique, est la capacité de certains génotypes à maintenir un état hydrique favorable. Ce maintien de la turgescence des tissus entretient l'activité métabolique de la plante et donc notamment une activité photosynthétique appréciable. Nous l’avons pu estimer à partir de l'accumulation de matière sèche dans le grain par rapport à celle remobilisée de la tige vers le grain. Ainsi, dans le premier cas, la capacité de remobilisation vers le grain des assimilats stockés dans le pédoncule, va être posée en termes d'efficacité d'accumulation de MSSE au cours de phases végétatives. Par contre pour le second cas, la remobilisation des réserves va être posée en termes de source complémentaire à l'activité photosynthétique.

\section{Remerciements}

Nous remercions infiniment le directeur de l'I.N.R.A. d'Alger (Algérie) Docteur Benbelkacem A.K. pour sa collaboration.

\section{References:}

Aissa N. et Radhouane L. Importance du statut hydrique et de l'indice chlorophyllien de la feuille drapeau du sorgho (Sorghum vulgare L.) dans l'élaboration du rendement grainée en présence de contraintes hydriques et salines. International Journal of Innovation and scientific Research, 2014, Vol 10, No 1, 111-117.

Bahlouli F., Bouzerzour H. et Benmahammed A. Effets de la vitesse et de la durée du remplissage du grain ainsi que l'accumulation des assimilats de la tige dans l'élaboration du rendement en grain (Triticum durum Desf.). Dans 
les conditions de culture des hautes plaines orientales d'Algérie. Biotechnol. Agron. Soc. Environ, 2008, 12 (1); 31- 39.

Barrs H.D. Determination of water deficits in plant tissues. In: water deficits and plant growth. Academic Press. Vol. I, 235-368.

Bidinger F., Muscrave RB. and Fisher RA. Contribution of stored preanthesis assimilate to grain yield in wheat and barley. Nature, 1977, 270, 431-433.

Bingham J. varietal reponse in wheat to water supply in the field and male sterility caused by period of drought in a glasshouse experiment. Ann.Appl. boil., 1966, 57, 365-377. In: Elaboration du rendement du blé d’hiver en conditions de déficit hydrique. I.Etude en lysimètres (Debaeke P. Puech J. Calas ML.) Agronomie, 1996, 16, 3-23.

Blacklow W.M., Darshire B. and Pheloung P. Fructans polymerized and depolymerized in the internodes of winter wheat as grain filling progressed. Plant Sci. Lett. 1984, 36, 213-218.

Cornic G. Drought stress and hight light effects on leaf photosynthesis. In Baker N.R., Bowyer J.R., editors. Photoinhibition of photosynthesis: from molecular mechanism to the field. Oxford, UK: Bios Scientific Publishers, 1994 PP 297- 313

Debaeke P., Puech J. et casals M.L. elaboration du rendement du blé dur d'hiver en conditions de déficit hydrique. I. Etude en lysimètres. Agronomie, 1996, 16, 3-23.

El Fakhri M., Mahboub S., Benchekroun M., Nsarellah N. Grain filling and stem accumulation effects on durum wheat (Triticum durum Desf.) yield under drought «Nature and technology» Journal, issue N N 7, 2012.

Gallagher F.N., Biscoe PV. and Hunter B. Effect of drought on grain growth. Nature, 1977, 264, 541-542.

Gate P., Bouthier A., Casabianca H. et Deleens E. Caractères physiologiques décrivant la tolérance à la sécheresse des blés cultivés en France: Interprétation des corrélations entre le rendement et la composition isotopique du carbone des grains. Colloque tolérance à la sécheresse des céréales en zones méditerranéenne. Diversité génétique et amélioration variétale, Montpellier (France), Décembre 1992, 15-17.

Kahali L. Etude de quelques paramètres de l'élaboration du rendement chez le blé dur (Triticum durum Desf.) cultivé en conditions de déficit hydrique. Thèse de Magister, Université de Constantine 1998, 86p.

Kong L, Wang F, Feng B, Li S, Si J, Zhang B. The structural and photosynthetic characteristics of the exposed peduncle of wheat (Triticum aestivum L.): an important photosynthate source for grain-filling. BMC Plant Biol. 2010 Jul, 11;10:141. doi: 10.1186/1471-2229-10-141.

Kuhbauch W. and Thome V. Non structural carbohydrate pattern in the cell content of wheat stems during grain filling. Crop. Sci. 1989, 155, 253-260. 
Lewicki S.D. Evaluation des paramètres liés à l’état hydrique chez le blé dur (Triticum durum Desf.) et de l'orge (Hordeum vulgare L.) soumis à un déficit hydrique modéré, en vue d'une application à la sélection de génotypes tolérants. Thèse de doctorat, 1993, 87p.

Loss SP. and Siddique KHM. Morphological and physiological traits associated whoth wheat yield increases in Mediterranean environment. Adv. Agron. 1994, 52, 229-276.

Maghsoudi Moud A. and Islami M. The effect of water stress on remobilization of pre-anthesis stores assimilates to grains in wheat. J.Plant Physiol. and Breeding. 2011, 1(1): 25-38.

Nachit MM. and Ketata H. Selection of mopho-physiological traits for abiotic streses resistance in durum wheat (Triticum durum). PhysiologyBreeding of winter Cereal for Stressed Mediterranean Environments. Colloques de l'INRA, 1991, No 55, 391-400.

Pandey P.C. Kaim MRS., Singh S.S., Singh G.P., Joshi D.K., Verma A.P.S. Post anthesis stem remobilization in new plant type wheat. Wheat Information Service, 2002, Number 95: 41- 42

Pheloung PC. and siddique KHM. Contribution of stem dry matter to grain yield in wheat cultivars. Aust.J. Plant Physiol. 1991, 18, 53-64.

Sanchez- Bragado R., Molero G., Reynolds MP., Araus JL. Relative contribution of shoot and ear photosynthetic to grain filling in wheat under good agronomical conditions assessed by differential organ $\delta 13 \mathrm{C}$. J. Exp. Bot. 2014, doi: 10.1093/jxb/eru 298.

Sinclair T.R., and Ludlow M. Who taugt plants thermodynamics? The unfulfilled potential of plant water potential of plant waterpotentiel. Aust.J. Plant physiol., 1985, 12, 213-217.

Slama A., Bensalem M., Ben naceur M.et Zid E. Les céréales en Tunisie: Production, effet de sécheresse et mécanisme de résistance. Sécheresse, 2005, Vol. 16 (3) (225- 229).

Triboi E., Rousset M. et Lemercier E. Elaboration du poids du grain. Agronomie, 1985b 11, 239-246.

Sofield L., Wardlaw I. F., Evans L.T. and Zee S.Y. Nitrogen, phosphorus and water contents during grain development and maturation on wheat. Aust.J. plant Physiol. 1977, 4; 799-810.

Yang J. and Zang J. Grain filling under soil drying. New Phytol. 2006, 169 (2): 223-236.

Zouaoui A., et Bensaid R. (2007). Détermination des exigences climatiques du blé dur (Triticum durum Desf. Var.Mohamed Ben Bachir) en zones semi arides. Cahier d'études et de recherche francophones / Agriculture, 2007, Vol. 16, N6, 469-79. 